\title{
Avoidance of Hidden Terminal \& Exposed terminal problem Using Directional MAC Protocol
}

\author{
Khushboo Agarwal and Vikas Sejwar \\ Department of CSE\&IT, \\ Madhav Institute of Technology and Science, Gwalior 474005 \\ ka.agarwal5@gmail.com
}

\begin{abstract}
The mobile Adhoc network is deployed in the environment where traditional wired network cannot be established due to its required features and their limitation. Use of directional antenna is one of the most promising technique for the high speed wireless network such as IEEE 802.11.Proposed a new directional MAC protocol to address the Directional Hidden terminal problem and Directional Exposed Terminal Problem.
\end{abstract}

Keyword: $A O D V$, MANET, transmission power, $C B R$

\section{Introduction}

In current scenario the uses of wireless communication are increases rapidly such as cellular communication, Mobile adhoc network etc. Wireless network are those,transmit information in a form of electromagnetic wave through the air the wireless networks are classified as Infrastructure less wireless network and Infrastructure dependent wireless network. Adhoc networks are comes in a category of wireless network that depend on multihop wireless radio communication with infrastructure less network.A MANET is a collection of mobile user that communicates over bandwidth constrained links ". It is a self-deployable network where nodes are mobile in nature and the no of node available at any instances can be changes dramatically. The routing decision and other activities are more complex due to the absence of any central coordinator. In the network topology, each device is termed as a node and the virtual connectivity among each node is termed as the link. There exists a link among the nodes when they are within the transmission range of each other. Nodes are organized themselves to transmit any message to other node by deploying a multihop communication if direct virtual link is not present.Most of the MAC layer protocol present in literature, deployed in a Omnidirectional anteena. Omnidirectional antenna cannot fully exploit the feature of the adhoc network. Due to this reason MAC protocol with directional antenna gets more interest of researcher.Directional antennahave various advantage over omni directional antenna in Adhoc networking. Directional antenna improve the spatial reuse, can provide longer transmission range even in the presence of lower transmission power.

\section{Related Work}

Most of the work on medium access of MANET has been done in the context of extending carrier sense multiple access/collision avoidance( CSMA/CA) to work with directional antenna.Several protocols have been defined in literature to tackle the directional hidden terminal problem and directional exposed terminal problem, most of them are based on the Distributed Coordination function (DCF), which are rely on RTS/CTS exchange to avoid the interference[3,4,7,16]. Nasipuri et al recommended one of the initial Directional MAC protocol, which utilize the Omni directional RTS and Omni directional CTS, but it is assume that the range of the directional transmission is 
same as the Omni directional transmission. Overhearing is one of the major problem in this case ,bad spatial reuse causes of overhearing [3].Y.Ko et alproposed a variation in traditional IEEE 802.11 DCF[1].Using additional hardware (i.e. GPS) tofind the location. But this DMAC protocol suffer from the collision at the sender side [4].Takai et al recommend the idea of DVCS ( Directional virtual carrier sensing) They uses a concept AOA(angle of arrival ) cache mechanism, whenever any node hear any signal from the neighboring node it estimate the AOA cache .This AOA cache information further use by the node when it become a source node for any data packet.They also proposed each node maintain a DNAV(Directional Network Allocation Vector),it use by the node for virtual carrier sensing[7].S.Motegi et al,Extend the method [30] for directional antenna, to deal with the hidden node problem. Author proposed the method to mitigate the directional hidden terminal problem known as DATA-frame fragmentation and short Busy Advertisement Signal (SBA-DMAC)[15].Takata et al,proposed a method, named as Directional MAC with Deafness Avoidance (DMAC/DA). This protocol simply a tradeoff between the deafness and WTS (Wait to send) additional control frame. After the successful handshaking of RTS -CTS, WTS (wait to send) frame is transmitted by the sender and the intended receiver to avoid the deafness[16]. In [5,6,12,27] propose a circular-DMAC to address the neighbor location problem.Korakis et al, propose the circular RTS MAC(CRM) protocol,it is the first protocol with circular transmission. In this protocol transmitter transmit RTS frame in a circular fashion by which the transmitter can able to scan the entire area and all the neighbor nodes can know about the upcoming transmission. With Circular RTS each node can be able to maintain the location information in the location table.There is no need to transmitter, to know the direction of the receiver and receiver can recognize the direction of the transmitter by using selective diversity. In this scheme transmission is done CRTS/DCTS/DATA/ACK [ 5].Jakllari et al [6], proposed the Circular RTS and CTS MAC(CRCM) protocol, It is a extension of CRM[5] .T.Korakis et al [12],Author proposed circular Directional RTS-MAC (CDRMAC)protocol, which represent efficiency in a static environment .In CDR-MAC protocol Circular RTS frame is transmitted as[5],to inform all the neighbor about upcoming transmission. Yihu li et al[27],proposed MAC protocol to avoid deafness and Collision problem named as directional MAC with deafness avoidance and collision avoidance(DMAC-DACA), in proposed protocol node transmit several Sweeping RTS/CTS packets anticlockwise to inform the neighbors about the upcoming transmission.Some directional MAC protocol present in the literature using the additional signal( Tone signal) to deal with the Hidden terminal and Exposed terminal problem[1720].

\section{Directional Hidden Terminal \& Exposed Terminal Scenario}

In this section we analyze the various scenarios in which Directional Hidden Terminal \& Exposed Terminal problem would occur. Usually Hidden terminal problem is causes of repetition of packet and Exposed terminal problem causes of not able to send a packet.However, Hidden Terminal problem refer to the collision of packet at the receiving node due to the simultaneous transmission of those node that are not within the direct transmission range of the sender, but are within the transmission range of the receiver. Collision occurs when both node transmit packet at the same time without knowing about the transmission of each other[10].There are two kind of Hidden Terminal problem

\section{Due to unheard DRTS/DCTS}

In the fig 1 , when communication in between node $\mathrm{A}$ and $\mathrm{B}$ is in progress, node $\mathrm{C}$ send DRTS to node D, this DRTS cannot hear by the node A, because node A beam formed in the direction of node B. Now when communication between node $\mathrm{C}$ and $\mathrm{D}$ is in progress node $\mathrm{A}$ finished its transmission to node $\mathrm{B}$ and want to communicate with 
node $\mathrm{C}$, send DRTS to node $\mathrm{C}$, this DRTS frame is collide with the ingoing transmission between node $\mathrm{C}$ and $\mathrm{D}$.
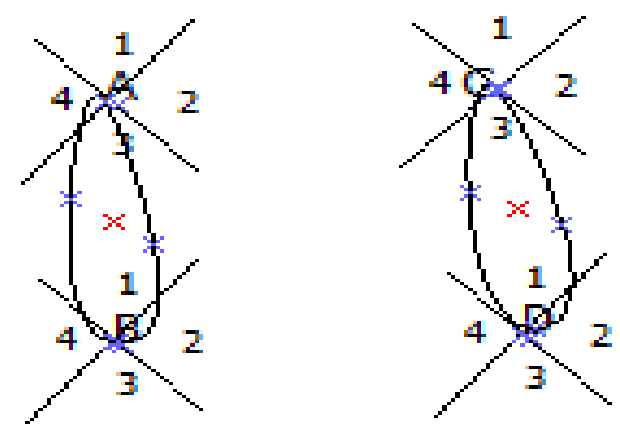

Figure 1. Hidden Terminal Problem Due to Unheared RTS/CTS

\section{Due to asymmetric Gain}

In fig2 Node $\mathrm{C}$ have data to node $\mathrm{D}$, node $\mathrm{C}$ sense the channel and send DRTS to node D,node D ack with DCTS. Node A is far away from node C,so it cannot receive this DRTS .If node $A$ want to communicate with node $D$, it sense the channel omnidirectionally, it cannot detet the ongoing transmission between node $\mathrm{C} \& \mathrm{D}$ due to $\mathrm{G}_{\mathrm{o}}<<\mathrm{G}_{\mathrm{d}}$. collision can occur. $\left(\mathrm{G}_{\mathrm{o}}\right.$ isthe Gain of omni directional antenna, $\mathrm{G}_{\mathrm{d}} \mathrm{isthe}$ Gain of directional antenna)
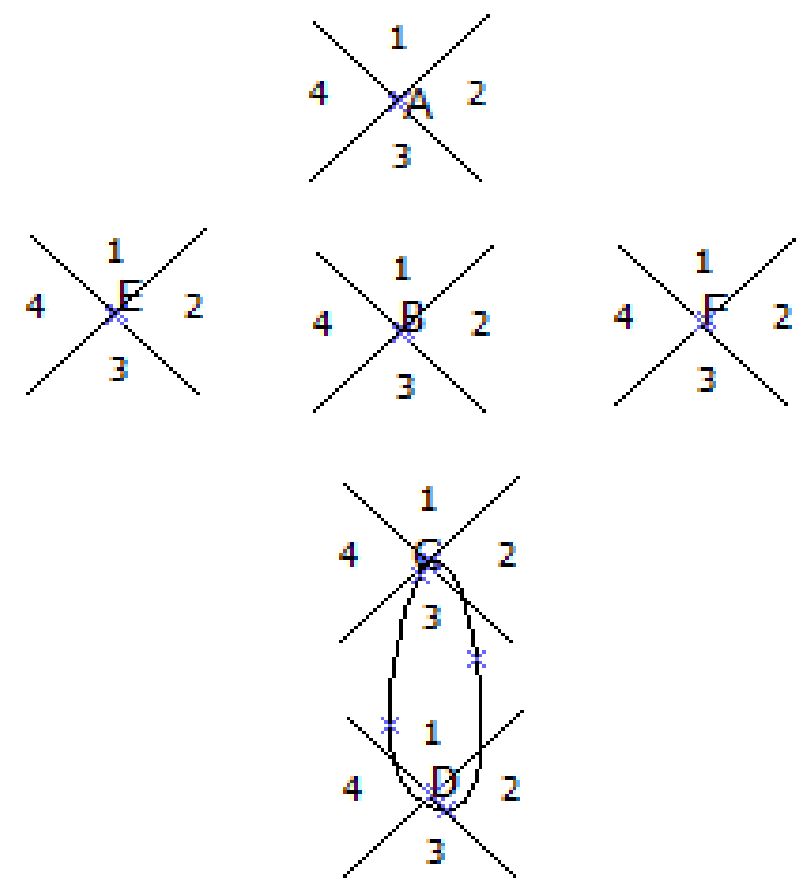

Figure 2. Hidden Terminal Problem Due to Asymmetric Gain

\section{Exposed Termial Problem}

The exposed terminal problem refer to the inability of a node, Which is blocked due to transmission by a nearby transmitting node ,to transmit another[10].In fig 3, Beam 3/ node $\mathrm{A}$ is directed toward beam $1 /$ node $\mathrm{B}$ and beam $1 /$ node $\mathrm{D}$. If node $\mathrm{A}$ have a data to 
node $\mathrm{D}$,it send DRTS to node $\mathrm{D}$, this DRTS packet is also received by node $\mathrm{B}$ because beam 1 node $\mathrm{B}$ is directed toward the beam $3 /$ node $\mathrm{A}$. If node $\mathrm{B}$ want to communicate with node $\mathrm{C}$, it deny the transmission while beam $4 /$ node $\mathrm{B}$ is free i.e. node A exposed their transmission.

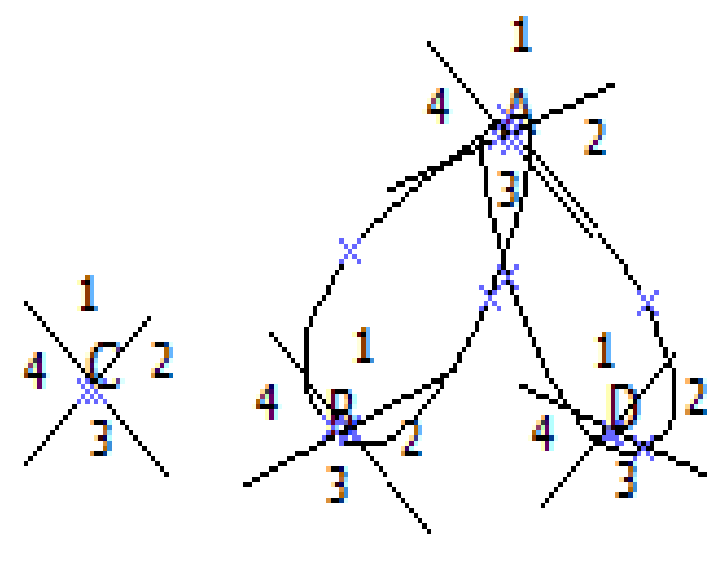

Figure 3. Exposed Terminal Problem

\section{Proposed Protocol}

In this section, we describe our Directional MAC protocol to solve the directional hidden terminal problem and directional exposed terminal problem.

In our proposed directional MAC protocol, all packets are transmitted directionally to fully exploit the directional antenna. To solve the neighbor location problem[20], proposed directional MAC protocol transmits the DRTS packet along with the positional information. This positional information is used by the neighbor node for further communication. Handshaking in this protocol is proposed to be using beam in sequence i.e. starting from the beam 1 to beam 4 even if the intended node is in beam 1-3.This will also take time outinto consideration depending upon the delay between considered nodes/network.

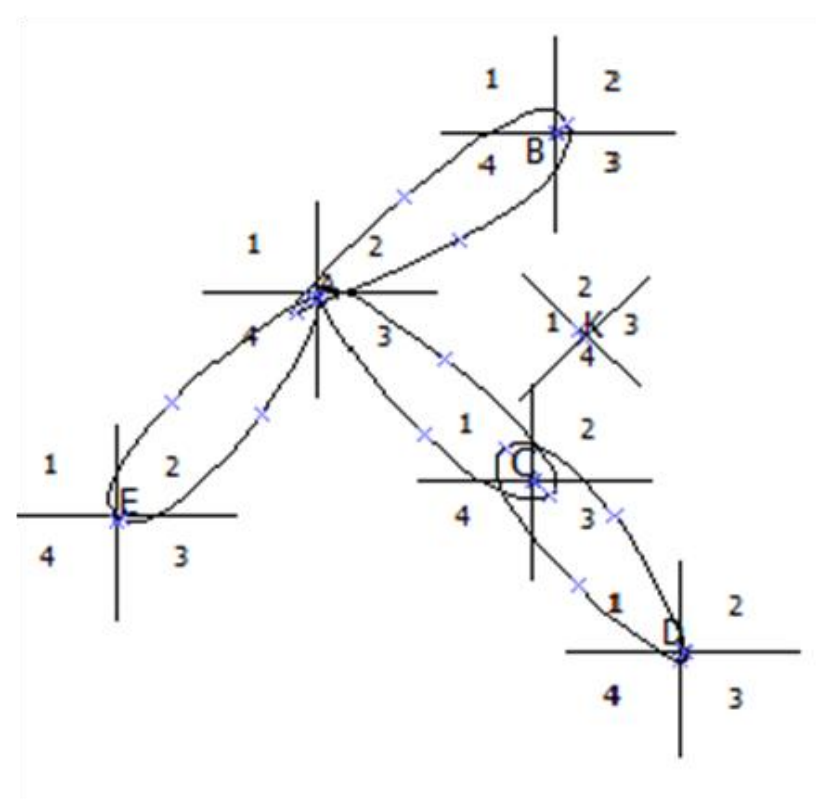

Figure 4. 
Consider the fig 4, assume that all the nodes are initially idle, they are listening the medium omnidirectionally. Also assume that node A transmit directionally only node B, $\mathrm{C}, \mathrm{K}$ and $\mathrm{E}$ able to receive signal while they are in their omnidirectionalmode. However communication is take place betweennode $\mathrm{A}$ and $\mathrm{C}$, if the beam of both the nodes are pointing to each other.With proposed DMAC communication between node $\mathrm{A}$ and $\mathrm{C}$ is possible as

ALGORITHM 1(Proposed DMAC)

- Node send a DRTS packet with positional information and wait for a predetermine time.

- All the neighbor node, who are able to receive this DRTS packet ,store location information of transmitting node for further communication.

- If node receives clearance information (DCTS) packet in this predetermine time then it send data packet directionally.

- Intended receiver response with ACK packet.

All nodes are initially idle, node A receive data packet for node $\mathrm{C}$ from upper layer. Node A sense the channel, and transmit the DRTS with positional information from all the beam one by one,start with beam 1 . Howeverall the neighbors of node $\mathrm{A}$ is aware about the upcoming transmission and the position of node A. Beam 3/node A is pointing toward the beam 1/node $\mathrm{C}$ and beam 1/node $\mathrm{K}$, node ADRTS packetwith positional informationthrough beam 3 is received by node $\mathrm{C}$ as well as node $\mathrm{K}$, node $\mathrm{K}$ update this positional information of node $\mathrm{A}$ for further reference and node $\mathrm{C}$ is response with DCTS packet , after the clearance of node $\mathrm{C}$ actual data transmission is begin.

\section{Implementation Details}

Here describe some implementation detail about the protocol. In proposed D-MAC, DRTS packet is transmitted using beam in sequence, it required some necessary change in some time periods that have been defined in 802.11[5],to support our protocol.Suppose communication is proceed in between node A and node C. node A transmit DRTS packet from all the beam one by one. DCTS is transmitted by the node C Just after the DRTS transmission is done by all the beam of node $\mathrm{A}$, time $\mathrm{T}$ is

$\mathrm{T}=\left(4-\mathrm{A}^{\prime}\right.$ s beam no)*DRTS transmission time + SIFS

Consider the fig 4 , node $\mathrm{C}$ receive DRTS with beam no 3 of node $\mathrm{A}$, then time $\mathrm{T}$ is

\section{Simulation}

To simulate the performance of our proposed DMAC, use Qualnet 5.2 simulator and perform simulation for various scenarios. 


\section{Packet drops due to retransmission limit}

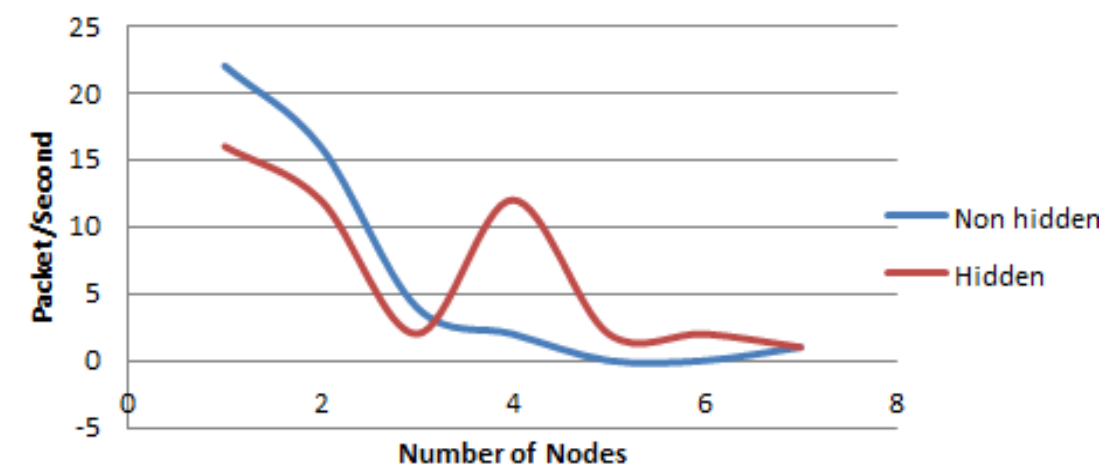

Figure 5.

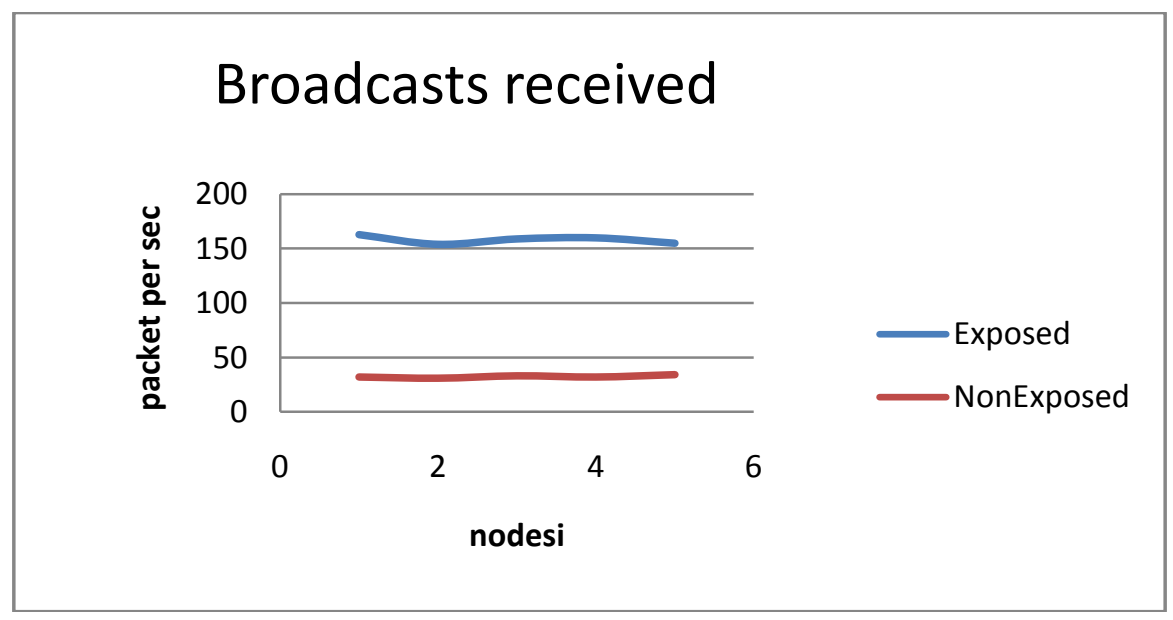

Figure 6.

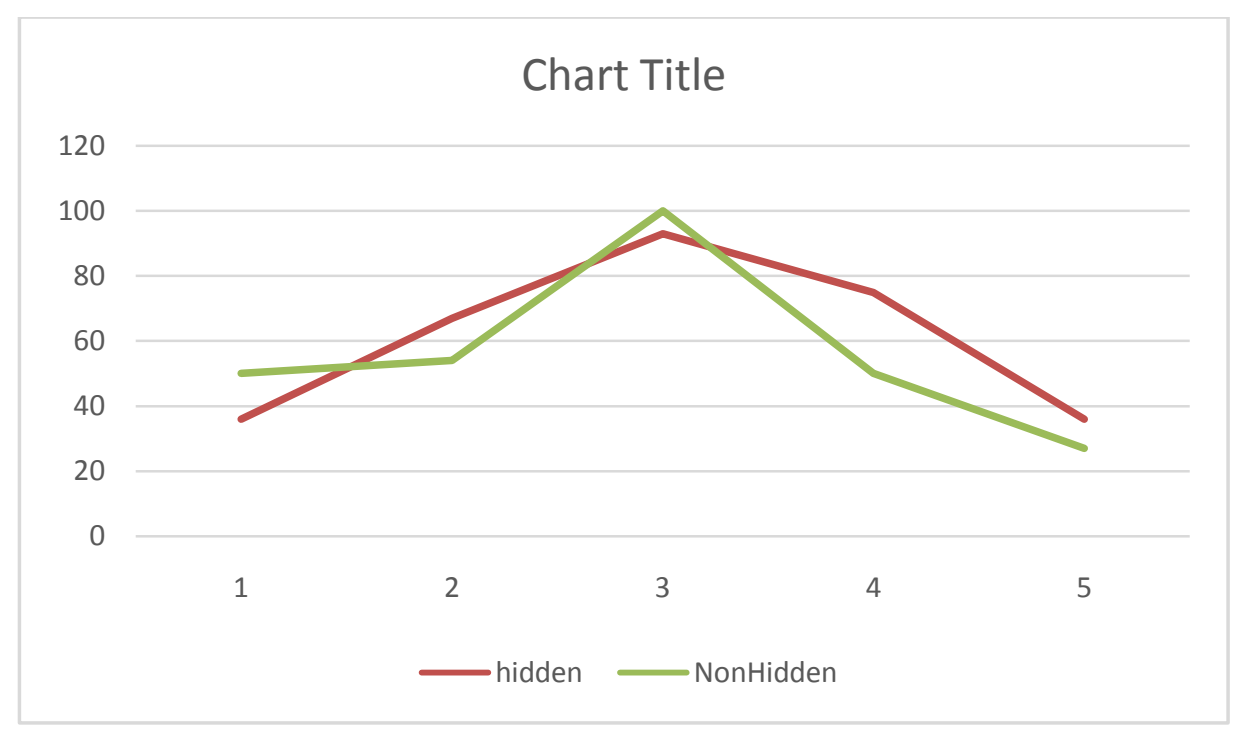

Figure 7. 


\section{References}

[1] ANSI/IEEE Std 802.11, "Wireless LAN medium access control (MAC) and physical layer (PHY) specifications", (1999).

[2] J.D.Kraus , R.J. Marhefka," Antennas For All Application”, TMH Third Edition.

[3] A. Nasipuri, S.Ye, J. You, and R. E. Hiromoto, "A MAC Protocol forMobile Ad Hoc Networks Using Directional Antennas", IEEE WirelessCommunications and Networking Conference (WCNC), (2000); Chicago, Illinois.

[4] Y. Ko, V. Shankarkumar, and N. Vaidya, "Medium Access Control Protocols Using Directional Antennas in Ad Hoc Networks", IEEEInternational Conference on Computer Communications (INFOCOM), (2000); Tel Aviv, Israel.

[5] T. Korakis, G. Jakllari, and L. Tassiulas, "A MAC protocol for full exploitation of Directional Antennas in Ad-hoc Wireless Networks", ACM International Conference on Mobile Computing and Networking (MobiHoc) , (2003); Annapolis, Maryland.

[6] G. Jakllari, I. Broustis, T. Korakis, S. V. Krishnamurthy and L. Tassiulas, "Handling Asymmetry in Gain in Directional Antenna Equipped Ad Hoc Networks", IEEE International Symposium on Personal,Indoor and Mobile Radio Communications (PIMRC), (2005),; Berlin,Germany.

[7] M. Takai, J. Martin, A. Ren, and R. Bagrodia, "Directional Virtual Carrier Sensing for Directional Antennas in Mobile Ad Hoc Networks", ACM International Symposium on Mobile Ad Hoc Networking andComputing (MobiHoc) , (2002); Lausanne, Switzerland.

[8] T. Elbatt, T. Anderson, \& B. Ryu, "Performance evaluation of multiple access protocols for ad hoc networks using directional antennas," In Proc. WCNC, (2003); NewOrleans, LA.

[9] R. Choudhury, X. Yang, R. Ramanathan, and N. Vaidya, "Using Directional Antennas for Medium Access Control in Ad Hoc Networks", ACM International Conference on Mobile Computing and Networking (Mobicom), (2002); Atalanta, Georgia.

[10] S. Kushwah, GS Tomar, Sarita Singh Bhadoria, "Analysis of DMAC Protocols in Wireless Ad hoc Networks", International Journal of Communication Systems and Network Technologies, vol.1, no. 2, (2012), pp 97-103.

[11] R. Ramanathan, J. Redi, C. Santivanez, D. Wiggins, and S. Polit,“Ad Hoc Networking with Directional Antennas: A Complete SystemSolution”, IEEE J. Sel. Areas Commun. , vol. 23, no. 3 ,(2005), pp. 496506.

[12] T. koraris, G. jakllari and L. Tassiulas, "CDR-MAC:AProtocol for Full Exploitation ofDirectional Antennas in Ad Hoc Wireless Networks", IEEE Transaction on mobile computing, (2008).

[13] N. Fahmy and T. Todd, "A Selective CSMA Protocol with Cooperative Nulling for Ad Hoc Networks with Smart Antennas”, IEEE Wireless Communications and Networking Conference (WCNC), (2004); Atlanta, Georgia.

[14] M. K. Dholey and A. Khatun, "Reduction of Blockage Node and hidden Terminal Problem of CSMA/CA MAC Protocol USING Directional Antenna", $2^{\text {nd }}$ IEEE International Conference on Parallel, Distributed and Grid Computing, (2012).

[15] J. C. Mundarath, P. Ramanathan, and B. D. V. Veen, "NULLHOC : A MAC Protocol for Adaptive Antenna Array Based Wireless Ad Hoc Networks in Multipath Environments", IEEE Global Telecommunications Conference (GLOBECOM), vol. 5, (2004); Dallas, Texas.

[16] S. Motegi, H. Sekiya, J. Ma, K. Sanada and S. Sakata, "A directional MAC Protocol with the DATAframe fragmentation and short Busy Advertisement Signal for Mitigating the Directional Hidden Node Problem", IEEE International Symposium on Personal, Indoor and Mobile Radio Communications (PIMRC), (2012).

[17] M. Takata, M. Bandai, and T. Watanabe, "A MAC Protocol with DirectionalAntennas for Deafness Avoidance in Ad Hoc Networks", IEEEGlobal Telecommunications Conference (GLOBECOM), (2007); Washington, USA.

[18] Z. Huang, C. Shen Z., C. Srisathapornphat, and C. Jaikaeo,"A Busy- Tone Based Directional MAC Protocol for Ad Hoc Networks,"in IEEE Military Communications in Conference (Milcom), vol. 2, Anaheim California, October 2002

[19] Y. Li, M. Li, W. Shu, and M.-Y. Wu, "FFT-DMAC: A Tone BasedMAC Protocol with Directional Antennas", IEEE Global Telecommunications Conference (GLOBECOM), (2007); Washington, USA.

[20] G.S. Tomar\& R.S. Tomar, "Position Based Routing For Mobile Ad Hoc Networks", International Conference UKSIM, (2008), pp. 555-560.

[21] H.- N. Dai, K. W. Ng, and M. Y. Wu, "A Busy-Tone based MAC Scheme for Wireless Ad b//Hoc Networks using Directional Antennas", IEEE Global Telecommunications Conference (GLOBECOM), (2007); Washington, USA.

[22] R. Rashmi, G. Dadahzadeh, E. Jedari \& M.Maleki, "An Auxiliary Tone Based MAC Scheme for High Density Ad Hoc Networks with Directional Antennas", IEEE Asian Pacific Conference on Communication (APCC 2009), (2009).

[23] G. Jakllari, W. Luo, and S. V. Krishnamurthy, "An Integrated Neighbor Discovery and MAC Protocol for Ad Hoc Networks Using Directional Antennas", IEEE Trans. Wireless Commun., vol. 6, no. 3, (2007), pp. 11-21. 
[24] J.-J. Chang, W. Liao and T.-C. Hou, "Reservation-Based Directional Medium Access Control (RDMAC) Protocol for Multi-hop Wireless Networks with Directional Antennas" IEEE International Conference on Communications (ICC), (2009), Dresden, Germany.

[25] W. Na, L. Park and S. Cho, "Deafness - aware MAC protocol for directional antennas in wireless ad hoc networks", Elsevier, (2014).

[26] S. Kushwah, S. S. Bhadauria, G.S. Tomar, "Deafness avoidance Protocols in Wireless Ad hoc Networks", International Journal of Communication Systems and Network Technologies, vol.1, no. 2, (2012), pp 97-103.

[27] Y. Li and A. Safwat, "On wireless Adhoc networks with directional antenna ;Efficient collision and deafness avoidance mechanism", Hindwai publicing corporation EURASIP journal on wireless communication and networking, (2008).

[28] G.S. Tomar, "Position Based Routing algorithm For Mobile Ad Hoc Networks", International Journal of Simulation- Systems, Science and Technology, vol. 10, no.1, (2009), pp 10-15.

[29] H. Zhai and Y. Fang, "A solution to hidden terminal problem overa single channel in wireless a hoc networks," IEEE Military Communications Conference MILCOM, (2006).

[30] A. P. Subramanian, S. R. Das, "Addressing deafness and hidden terminal problem in directional antenna based wireless multi-hop networks", Journal of Wireless Networks, vol. 16, no. 6, (2010), pp 15571567. 